\title{
EASL-EASD-EASO Clinical Practice Guidelines for the management of non-alcoholic fatty liver disease: disease mongering or call to action?
}

\author{
Elisabetta Bugianesi ${ }^{1}$ \\ Received: 29 February 2016 / Accepted: 1 March 2016/Published online: 7 April 2016 \\ (C) Springer-Verlag Berlin Heidelberg 2016
}

Keywords Cost-effectiveness $\cdot$ Fatty liver $\cdot$ Guidelines · Screening

$\begin{array}{ll}\text { Abbreviations } \\ \text { ALT } & \text { Alanine transaminase } \\ \text { AST } & \text { Aspartate transaminase } \\ \text { FDA } & \text { Food and Drug Administration } \\ \text { FLI } & \text { Fatty Liver Index } \\ \text { HCV } & \text { Hepatitis C virus } \\ \text { NAFL } & \text { Non-alcoholic fatty liver } \\ \text { NAFLD } & \text { Non-alcoholic fatty liver disease } \\ \text { NASH } & \text { Non-alcoholic steatohepatitis }\end{array}$

Traditionally considered the hepatic manifestation of the metabolic syndrome, non-alcoholic fatty liver disease (NAFLD) is one of the most common causes of chronic liver disease worldwide, and has dramatically increased in concert with the epidemics of both adult and childhood obesity and type 2 diabetes mellitus [1, 2]. NAFLD includes two distinct

Elisabetta Bugianesi was invited by Diabetologia and the European Association for the Study of the Liver to discuss the practical, ethical and economic implications of the EASL-EASD-EASO Clinical Practice Guidelines for the management of non-alcoholic fatty liver disease. The views expressed are those of the author and not those of the society.

Elisabetta Bugianesi

elisabetta.bugianesi@unito.it

1 Division of Gastroenterology, Department of Medical Sciences, University of Torino, AOU Città della Salute e della Scienza, Corso Dogliotti 14, 10126 Torino, Italy conditions with different histological features and prognoses: non-alcoholic fatty liver (NAFL) and non-alcoholic steatohepatitis (NASH); steatohepatitis and significant fibrosis are considered harbingers of adverse hepatic outcomes in individuals with NAFLD and are associated with an increased risk for morbidity and mortality through hepatic (progression to end-stage liver disease and hepatocellular carcinoma) and non-hepatic (mainly cardiovascular) complications [3]. Approximately $30 \%$ of adults and $10 \%$ of children in Western countries have NAFLD, but only a minority, albeit significant, experience associated morbidity. What determines the progression to NASH and beyond is not clear. NAFLD is characterised by substantial inter-patient variability in terms of severity and rate of progression, with fairly rapid disease progression in certain subgroups [4]. The diagnosis of NASH is at present based on histological assessment by liver biopsy. The procedure is invasive and has associated risks, even if they are small. However, to date, both the US Food and Drug Administration (FDA) and the European Medicines Agency require the demonstration of improvements in liver histological endpoints for the market approval of any new pharmacological treatment for NASH. Specifically, the FDA does not recognise NAFL as a treatment indication, and any new treatment should focus on improving NASH. Although there are no therapeutic interventions currently approved for use in $\mathrm{NASH}$, the clinical trial landscape will experience a surge of innovation and exponential growth over the coming years.

In this context, the European Association for the Study of the Liver (EASL), the European Association for the Study of Diabetes (EASD) and the European Association for the Study of Obesity (EASO) joint guidelines for the management of NAFLD [5] represent an important step forward, first because they have been developed by organisations with a shared awareness of the multidisciplinary nature of this disease, and second because they set up a common ground to optimise the 
management of patients and fill the knowledge gaps on the course of hepatic and extrahepatic comorbidities. In addition, the authors explain that one aim of the guidelines is to improve patient care and awareness of the importance of NAFLD and to assist stakeholders in the decision-making process by evidence-based data.

First of all, what patients need to know about this disease? Patients, particularly obese and diabetic individuals, need to be clearly informed that they have a risk of liver-related complications and death driven by NASH, similar to how they are informed of cardiovascular, metabolic and kidney complications..

Ultrasound-diagnosed NAFLD is not associated with increased liver-related mortality, but in NASH liver disease is the third most common cause of death after cardiovascular disease and cancer, and the risk is increased tenfold compared with that for the general population [2]. Indeed, NASH resembles hepatitis $\mathrm{C}$ virus-related chronic liver disease in terms of its insidious onset and indolent course, often diagnosed at the time of cirrhosis, particularly in patients with type 2 diabetes. A recent study in Hong Kong reported that $73 \%$ of the diabetic patients at primary care and hospital clinics had fatty liver and $18 \%$ had increased liver stiffness by transient elastography suggestive of advanced fibrosis independently of liver enzymes [6].

Information is an ethical duty and it is the first step toward prevention, as patients should avoid other risk factors for liver disease, such as occasional alcohol abuse or potentially hepatotoxic drugs. While this can translate into a case-by-case screening for NASH in adults, where the liver-related risk must always be weighed up against the cardiovascular risk, children represent the most vulnerable population that does have the potential to develp the full spectrum of complications associated with NASH [7] and hence deserves an indepth approach, according to what suggested by the guidelines.

That said, what sets NAFLD apart from other common liver diseases is the sheer volume of patients with this disease and the lack of a definite therapeutic modality. In this context, the major focus of clinical care for patients with NAFLD is discerning those at highest risk for the complications of advanced liver disease. The guidelines recommend that individuals with obesity or any component of the metabolic syndrome should have an ultrasound and steatosis biomarkers and liver enzymes measured, particularly patients with type 2 diabetes, for whom the presence of NAFLD should be looked for irrespective of liver enzyme levels [5]. On the one hand, this would include the full spectrum of NAFLD in the real life setting, the most important piece of information missing so far, but on the other, this would represent an extreme challenge in terms of the resources of national heathcare systems. A cost-utility analysis of NASH screening is hampered by uncertainties surrounding the non-invasive markers of liver damage and the lack of effectiveness data relating to the impact of treatment on fibrosis in patients with NASH. In an exploratory analysis on the use of non-invasive tests to determine referral to tertiary care [8], the analysis of the incremental cost per correct positive diagnosis found that most of the tests were dominated by liver biopsy; however, the analysis per correct negative diagnosis found that fibrosis 4 calculator (FIB-4) (high cut-off) and NAFLD Fibrosis Score (NAFLD-FS) (high cut-off) had an incremental costeffectiveness ratio of below $£ 1$, while the one for biopsy was $£ 145.39$. Another cost-utility analysis in middle-aged diabetic individuals [9], which compared a screening strategy for NASH (one-time liver ultrasound in all and liver biopsies in those with fatty liver) vs no screening, found that the first led to a $12.9 \%$ reduction in incident cirrhosis and an $11.9 \%$ decrease in liver-related deaths, but was associated with 0.02 fewer quality-adjusted life years as a result of non-adherence to the treatment regimen because of side effects (i.e. weight gain associated with pioglitazone). The model suggests that screening for NASH is likely to be cost-effective only when medications with mild side effects, even with those with modest efficacy, become available. Although no such medication is currently available, there has been rapid development in this field, with over 200 studies listed on ClinicalTrials.gov and encouraging results from recent trials.

In the meantime, how can assessments be implemented, particularly in the primary care setting? First, the noninvasive measures of steatosis, such as the fatty liver index (FLI), can easily be calculated from clinical and biochemical data to screen for the presence/absence of NAFLD. Assessment of liver enzymes incurs additional costs, but not very different from those associated with screening for cardiovascular or kidney damage. Although abnormal liver function test (LFT) results are an indication for specialist referral, patients with normal LFTs can experience liver problems as well. However, the absolute value of alanine transaminase (ALT) can still be a prognostic indicator. In adults, the upper limit of 'normal' ALT has been reduced to $30 \mathrm{U} / 1$ for men and $19 \mathrm{U} / \mathrm{l}$ for women [10], while according to the Screening ALT for Elevation in Today's Youth (SAFETY) study [11], the commonly proposed cut-off values for an elevated ALT level should be reduced to $25 \mathrm{U} / 1$ in boys and $22 \mathrm{U} / 1$ in girls. Furthermore, the same easily available variables (clinical, anthropometric and laboratory) can be used in the two abovementioned scoring systems, FIB-4 and NAFLD-FS, to exclude severe NASH. The NAFLD-FS is calculated based on age, BMI, hyperglycaemia or diabetes, aspartate transaminase (AST)/ALT, platelets, and albumin (www.nafldscore.com), while the FIB-4 is based on age, AST, ALT and platelets [12]. The presence, duration and severity of obesity and type 2 diabetes, along with the presence of other risk factors (such as familial history of liver disease) or any signs and symptoms of chronic liver disease, are important indicators of possible NASH. 
What is the ultimate aim of these guidelines? Most importantly, to harmonise a myriad of different approaches, languages and thinking about this intriguing and potentially lethal liver disease. The bare truth is that we need to know more about NAFLD, as dramatically underlined by the lack of noninvasive biomarkers of NASH, which hampers clinical practice and seriously impedes drug development. The most successful disease management programmes are those where the pathogenesis and the natural history of the disease are well understood, but hepatologists see only the extreme end of the spectrum of NAFLD. We need to find a common ground where all the major players in the metabolic field can cooperate, sharing resources, clinical data and patient samples, to tackle this modern-day disease and translate 'disease mongering' into an effective way forward for the patients and for the healthcare systems.

Duality of interest The author declares that there is no duality of interest associated with this manuscript.

Contribution statement The author was the sole contributor to this paper.

\section{References}

1. Yki-Järvinen H (2014) Non-alcoholic fatty liver disease as a cause and a consequence of metabolic syndrome. Lancet Diabetes Endocrinol 2:901-910

2. Bhala N, Jouness RI, Bugianesi E (2013) Epidemiology and natural history of patients with NAFLD. Curr Pharm Des 19:5169-5176

3. Byrne CD, Targher G (2015) NAFLD: a multisystem disease. J Hepatol 62:S47-S64
4. Singh S, Allen AM, Wang Z, Prokop LJ, Murad MH, Loomba R (2015) Fibrosis progression in nonalcoholic fatty liver vs nonalcoholic steatohepatitis: a systematic review and meta-analysis of paired-biopsy studies. Clin Gastroenterol Hepatol 13:643-654

5. European Association for the Study of the Liver (EASL), European Association for the Study of Diabetes (EASD) and European Association for the Study of Obesity (EASO) (2016) EASLEASD-EASO clinical practice guidelines for the management of non-alcoholic fatty liver disease. Diabetologia. doi:10.1007/ s00125-016-3902-y

6. Kwok R, Choi KC, Wong GL et al (2015) Screening diabetic patients for non-alcoholic fatty liver disease with controlled attenuation parameter and liver stiffness measurements: a prospective cohort study. Gut. doi:10.1136/gutjnl-2015-309265

7. Nobili V, Svegliati-Baroni G, Alisi A, Miele L, Valenti L, Vajro P (2013) A 360-degree overview of paediatric NAFLD: recent insights. J Hepatol 58:1218-1229

8. Crossan C, Tsochatzis EA, Longworth L et al (2015) Costeffectiveness of non-invasive methods for assessment and monitoring of liver fibrosis and cirrhosis in patients with chronic liver disease: systematic review and economic evaluation. Health Technol Assess 19:1-409. doi:10.3310/hta19090, v-vi

9. Corey KE, Klebanoff MJ, Tramontano AC, Chung RT, Hur C (2016) Screening for nonalcoholic steatohepatitis in individuals with type 2 diabetes: a cost-effectiveness analysis. Dig Dis Sci 29: doi: 10.1007/s10620-016-4044-2

10. Prati D, Taioli E, Zanella A et al (2001) Updated definitions of healthy ranges for serum alanine aminotransferase levels. Ann Intern Med 137:1-10

11. Schwimmer JB, Dunn W, Norman GJ et al (2010) SAFETY study: alanine aminotransferase cutoff values are set too high for reliable detection of pediatric chronic liver disease. Gastroenterology 138: 1357-1364

12. European Association for the Study of the Liver, Asociacion Latinoamericana para el Estudio del Higado (2015) EASL-ALEH clinical practice guidelines: non-invasive tests for evaluation of liver disease severity and prognosis. J Hepatol 63:237-264 